\title{
Promoting Advanced Traveler Information Systems Among Cellular and Land-Line Phone Users SmarTraveler Experience in Boston
}

\author{
LARry S. Englisher, SusAn BREgman, StePHEN PePIN, AND \\ Alicia POWELl WiLsON
}

In 1993 the SmarTraveler advanced traveler information system (ATIS) was introduced to travelers in the greater Boston area as part of an operational test jointly funded by FHWA and the Massachusetts Executive Office of Transportation and Construction. The service offers real-time, route-specific travel information by phone for major highways and public transit. During the 3-year test period from January 1993 to January 1996 , service usage grew to nearly 60,000 calls per week, made by an average of more than 20,000 users. To achieve this growth, SmarTraveler was marketed to both cellular and land-line phone users using a variety of strategies over the course of the test. Evaluation and monitoring studies were commissioned to examine the degree to which users responded to SmarTraveler and the marketing program. The evaluation included extensive surveys of users and nonusers as well as tracking of calls and call frequency. The findings of the independent evaluation team on the success of various promotional efforts associated with the SmarTraveler operational test are presented. Overall, the marketing program aimed at the broad target market during the first 2 years appears to have been only partly successful in increasing the public's level of awareness of SmarTraveler, in convincing its target market of SmarTraveler's superiority to radio and TV travel reports, and in inducing trials. As a result of the second-year evaluation, changes were made to marketing and pricing during the third year of operation to target cellular telephone users. Further surveys were conducted to examine cellular telephone user response to these changes. SmarTraveler experienced a large increase in calls and users in the third year, especially in the cellular telephone market. Among the survey findings was that this market is quite price sensitive.

SmarTraveler is an advanced traveler information system (ATIS) that offers real-time, route-specific traffic and transit information to travelers in the Boston metropolitan area via telephone. An operational test of SmarTraveler was jointly funded by FHWA and the Massachusetts Executive Office of Transportation and Construction; it also received monetary contributions and in-kind services from the private sector equal to the federal share. SmarTraveler began 3 years of operational test service on January 13, 1993, following a 21/2month scale up period designated as Phase I. Service continues to operate after the test period under contract between Massachusetts Highway Department and the private operator, SmartRoute Systems. Phase II consisted of the first 15 months of operation and Phase III, the following 9 months. This paper presents findings on the impacts

L. S. Englisher and S. Bregman, Multisystems, Inc., 10 Fawcett Street, Cambridge, Mass. 02138. S. Pepin, Bureau of Transportation Planning and Development, Massachusetts Executive Office of Transportation and Construction, 10 Park Plaza, Boston, Mass. 02116. A. P. Wilson, Central Transportation Planning Staff, 10 Park Plaza, Boston, Mass. 02116. of the Phase III marketing program conducted during the period from April 1 through December 31, 1994, and the effect of marketing and privileges in the Phase IV during 1995.

\section{DESCRIPTION OF SMARTRAVELER OPERATIONAL TEST}

Callers to SmarTraveler can choose to hear a recorded report on one or more of 21 monitored highway segments or four public transportation services by entering a key code. The real-time reports include conditions, travel times, and anomalies such as accidents, but they do not typically give explicit alternative highway routes. Travel conditions are monitored from 5:30 a.m. to 7:00 p.m. Monday through Thursday, until 9:00 p.m. on Friday, and from noon to 7:00 p.m. on Sunday. Conventional phone users (referred to as landline callers) access the system by dialing (617) 374-1234. They pay no fee for the information they receive beyond the usual conventional telephone fees. Subscribers to the NYNEX cellular phone service simply dial $* 1$ and are not subject to charges of any kind. During the Phase III evaluation period, Cellular One subscribers could dial either the land-line phone number or *ST1; in either case, they had to pay the cost of the phone call. In July 1995 during Phase IV, however, arrangements were made to offer Cellular One subscribers the same access number and free calls as the NYNEX cellular users. The Phase II evaluation found that NYNEX cellular phone subscribers were the most avid users of SmarTraveler under typical weather conditions and that much of the traveling public was unaware of SmarTraveler and did not understand its advantages. Marketing the service was a key component of Phase III (the second year of operations) and was addressed in the evaluation of Phase III.

\section{PHASE III MARKETING PROGRAM ACTIVITIES}

The Phase III marketing plan was designed to accomplish three key objectives identified during Phase II as necessary for more rapid growth of SmarTraveler: $(a)$ to make the traveling public more aware of the service, $(b)$ to differentiate the service from other sources of travel information, and $(c)$ to improve the public's recollection of the service's land-line telephone access number. A key target group was those who currently listen to broadcast media traffic reports and news reports. 
To achieve these objectives, SmarTraveler implemented a diverse program involving many components and media, including television and radio ads and mentions; billboards, dioramas, posters, and other public displays; handouts, flyers, and direct mailings; and news articles. Figure 1 shows the number, frequency, and timing with which these components were implemented. The marketing program incorporated nearly $\$ 1$ million in in-kind contributions from public agencies and media outlets, about four times the value of SmartRoute Systems' out-of-pocket expenditures for marketing materials. In-kind contributions included radio and television time, billboards, variable message signs on the Massachusetts Turnpike, and MBTA (transit) car cards.

\section{IMPACTS OF MARKETING CAMPAIGN}

The marketing program was the major innovation introduced in Phase III; thus, assessing its effectiveness was a critical element of the Phase III evaluation. One can divide the SmarTraveler market- ing objectives into three stages: $(a)$ raising awareness among the target population; $(b)$ inducing program trial, in the form of a user call for information; and (c) retaining callers as regular users. Each of these is a useful evaluation criterion. This paper focuses on the first two stages, awareness and trial; information on retention is currently incomplete and is more appropriately the subject of future evaluation efforts. The observations and conclusions here are derived from the analysis of user surveys, target market surveys, and call count data. Nearly 1,000 user and 2,000 target market surveys were conducted during Phase III alone.

\section{Exposure to Media Advertising}

\section{User Exposure to Media Advertising}

Radio and TV ads constituted a large part of the marketing program, and about half of all users saw or heard the ads on each medium. New users (those users who said they first used SmarTraveler in

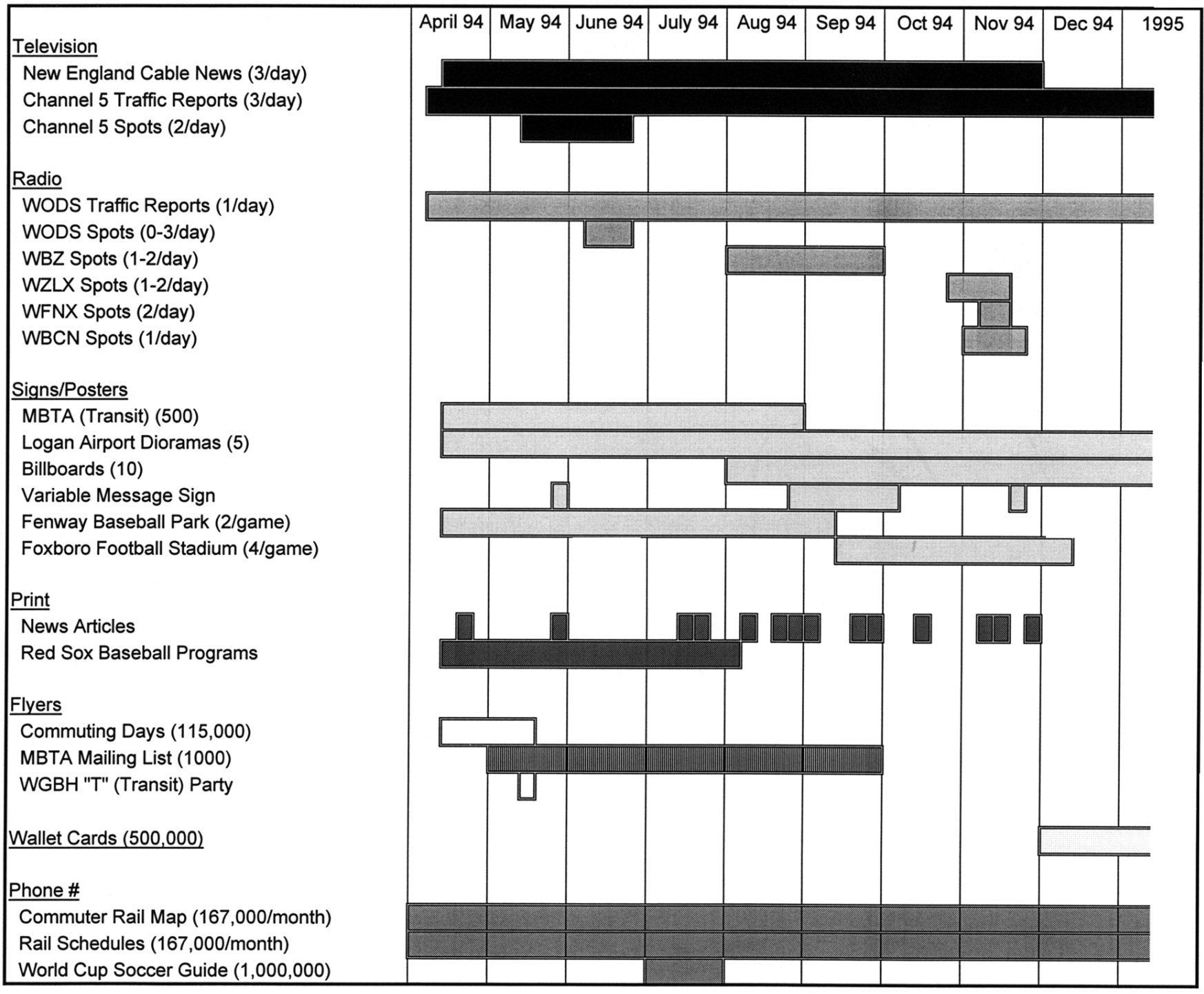

FIGURE 1 SmarTraveler Phase III marketing program timeline. 
Phase III) were somewhat more likely to have heard the radio ads aired on WBZ, the primary news and traffic information station, than the TV ads. The frequency of exposure to the radio and TV ads was similar among those who reported seeing or hearing them. A large share of new users as well as early users (those who first used SmarTraveler before April 2, 1994) reported hearing radio and TV ads six or more times.

\section{Nonuser Exposure to Media Advertising}

The majority ( 63 percent) of all nonusers (those who never had tried SmarTraveler) do not recall being exposed to any marketing mention or ad for the service. This appears to be related to their low rates of reliance on broadcast travel information, as recorded in the surveys. That is, most travelers are simply not information seekers. Those who listen to broadcast media traffic reports were more likely than other nonusers to have been exposed to the SmarTraveler marketing campaign. Thus, the campaign was successful in targeting this group. In fact, these individuals were found to listen to WBZ radio in particular, which was the primary station on which radio ads aired.

\section{Impact of Media Advertising}

Neither users nor nonusers found the radio or TV ads very memorable. About half of the users and more than two-thirds of nonusers who reported being exposed to the ads indicated that they did not recall the ads very well. Despite these findings, it is believed that the use of radio and TV spots is a fundamentally sound strategy. However, it is clear from the survey results that some adjustments in message content and media coverage will be required if radio and TV ads are to be more effective.

\section{Cellular Versus Land-Line Users}

NYNEX cellular telephone users were less likely than land-line users to have been influenced in any way by media advertising: 88 percent of the NYNEX users reported that the radio ads and 71 percent of NYNEX users reported that the TV ads had no influence (compared with 52 and 47 percent of the land-line users). This may reflect a greater reliance by NYNEX users on SmarTraveler than on radio reports. (Or it may mean that they were introduced to the service by other promotions and were less prone to further influence by the media campaign.) Even among land-line users, the influence of the broadcast media was limited. At least 40 percent of the land-line users who saw radio and TV ads did not remember them very well, and about half said that the ads did not influence them at all. Among those users who said they were affected by the radio ads, about half of both NYNEX and land-line users said the ads influenced them to call the first time (although the NYNEX group was too small to be significant). Among land-line users who were influenced, 29 percent said the ad reminded them to call and 12 percent said it reminded them of the phone number. The impact of the TV ad mirrors that of the radio ad.

Clearly, a significant percentage of the target population is not receiving, or perhaps failing to notice, information about SmarTraveler from the current broadcast media advertisements. The inability to reach these individuals may be one of several reasons that growth of the active calling population stalled during Phase III. Furthermore, perhaps those who do not listen to media traffic reports will not respond to marketing unless the message includes a clear and engaging portrayal of the benefits of SmarTraveler (e.g., travel time savings, reduced stress, increased ability to plan one's time, etc.).

\section{Awareness of SmarTraveler}

\section{Overall Level of Awareness}

The results of the target market surveys indicate that awareness of SmarTraveler in the target population did not increase much during Phase III. More than half (55 percent) of the target market (and 60 percent of the nonusers) were unaware of SmarTraveler after more than 20 months of operation and a continuous marketing campaign. Furthermore, as in Phase II, only 31 percent of the target market can be characterized as being truly aware of the service since more than one-third of those who said they were aware of SmarTraveler were not sure what it was. These findings indicate that the effect of the marketing campaign on the overall level of awareness of the target market was relatively small during Phase III.

Even though the overall level of awareness did not change significantly since Phase II, some additional nonusers became aware of SmarTraveler during Phase III and the marketing campaign was a significant factor in their awareness: at least 60 percent of the nonusers who indicated that they had become aware of SmarTraveler during Phase III cited radio and TV ads as the source responsible for their knowledge. In addition, as many as 22 percent of new users reported that their initial source of awareness was radio ads. (Although these findings appear to conflict with the conclusion that overall awareness of the target market did not increase substantially, they may be less reliable since respondents' ability to recall when they first learned about SmarTraveler is not infallible; questions about overall awareness are less subject to error and are therefore more reliable.)

\section{Knowledge of SmarTraveler Phone Number}

Most users surveyed knew the SmarTraveler phone number, and awareness increased among users between phases. This could have resulted from increasing frequency of use, from the marketing campaign, or both. NYNEX users were more likely than land-line users to know the SmarTraveler telephone number: 88 versus 61 percent. Of course, this familiarity most likely reflects the simplicity of the NYNEX number, which is $* 1$ instead of 374-1234. In contrast, nonusers had very limited knowledge of SmarTraveler's phone number. Whereas 71 percent of all users could correctly state the phone number, only 5 percent of the nonusers who were familiar with SmarTraveler could do so. Ignorance of the phone number is a serious impediment to both trial and use. The large number of users and nonusers who did not know the phone number despite the emphasis placed on this throughout the marketing program is significant.

\section{Sources of User Awareness}

A major finding of the user survey analysis is that the principal marketing program components responsible for the observed levels of first awareness among users are, collectively, the electronic broadcast media, both radio and television. However, excluding the 
Channel 5 TV mentions (during news traffic reports) and looking only at advertisements, the implied marketing effectiveness of the broadcast media is less encouraging. The apparent weakness of the ads in generating program awareness is at least partly explained by a low exposure rate of the advertisements to potential users. As described in the preceding section, 63 percent of all nonusers do not recall being exposed to any ad or marketing mention.

New users in Phase III first learned about SmarTraveler from three major sources: radio advertising, word of mouth, and NYNEX literature; television ads and Channel 5 mentions were slightly less effective. Taken together, the electronic media were the source of awareness for 45 percent of new users. While word of mouth played a significant role, it can be considered a by-product of the SmarTraveler service rather than a primary marketing component.

\section{Cellular Versus Land-Line Users}

Consistent with the marketing program goals, there is strong evidence that the marketing campaign successfully targeted land-line users. More of the Phase III land-line users (55 percent) became aware of SmarTraveler during Phase III than NYNEX users (44 percent). Figure 2 indicates that among land-line users, radio ads were the leading source of awareness, followed by word of mouth and the television ads. In contrast, most NYNEX users learned about SmarTraveler from NYNEX promotional literature (which consisted of a single collateral piece distributed at the end of Phase III) and word of mouth.

\section{Nonuser Awareness and Its Sources}

Fewer than half (41 percent) of nonusers surveyed during Phase III had any awareness of SmarTraveler. Actually, only 24 percent said they knew what SmarTraveler was; the others had just heard of it. Only 12 percent of all the nonusers surveyed said that they became aware after the Phase III marketing activities began in spring 1994.
(This number may be overstated if respondents were mistaken about when they first became aware of SmarTraveler.)

The marketing campaign was largely responsible for the awareness of individuals who reported becoming aware during Phase III. Radio and television ads each were responsible for 37 percent, while mentions on Channel 5 traffic reports played a smaller role, at 8 percent. Though it was so influential among users, it is interesting to note that word of mouth played a very small role (5 percent) in informing nonusers. This suggests that word of mouth is particularly effective in generating trials (rather than simply creating awareness).

Among all nonusers surveyed during Phase III, television played a larger role than radio in raising awareness. This success is in sharp contrast to the lesser effect of the television ads on the awareness of the SmarTraveler users. The fact that nonusers were more likely than users to become aware of SmarTraveler through television may suggest that the other marketing media were more able to target those with potential to become users or that the specific messages conveyed by the television ads raised awareness but failed to induce trials.

\section{Level of Understanding of SmarTraveler}

Although many nonusers appear to understand SmarTraveler's attributes, others still have several misconceptions about SmarTraveler and the information it provides. No improvement in these aspects of awareness occurred among the target market population since Phase II. Of particular concern, many nonusers still did not understand where SmarTraveler gets its information and how the service differs from media travel reports.

\section{Influence of Marketing on Trials of SmarTraveler}

Among current users, the most frequently named elements of the marketing program influencing trials were NYNEX literature (25 percent), radio and TV ads (15 percent each), and mentions

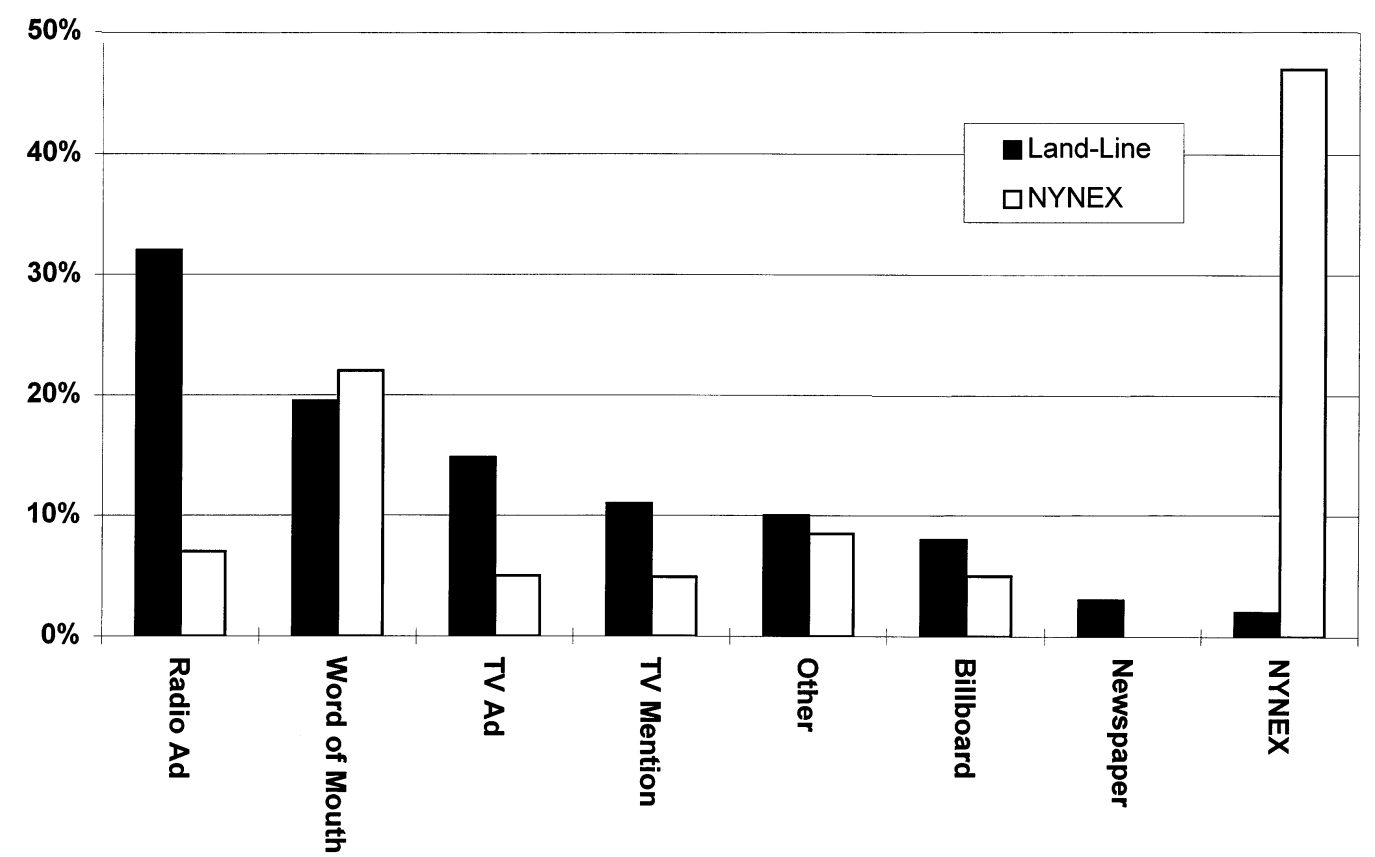

FIGURE 2 How users became aware of SmarTraveler. 
during Channel 5 news traffic reports (14 percent). A detailed analysis of the relationship between call counts and marketing activities appears to indicate an acceleration of the growth rate of total calls during the 3-month period in which the marketing program was intensifying. Total calls on selected days were compared with the number of radio ads that aired on the previous day. This analysis indicated that each additional radio ad airing correlated with an increase of 75 calls on the following day. Nevertheless, word of mouth, not a primary focus of the marketing campaign per se, was reported as an information source by more users (17 percent) than any other factor except the NYNEX literature. In fact, broadcast media components collectively had about the same total effect on trials of SmarTraveler over the entire period of operation as NYNEX literature and word of mouth combined. The nonbroadcast components of the marketing program (i.e., billboards, dioramas, newspaper articles, etc.) appeared to contribute relatively little to awareness or trial.

As Figure 3 clearly shows, broadcast media marketing was the primary influence on trials among land-line users, responsible for 65 percent of first trials, with word of mouth affecting another 15 percent. The influence of the media campaign on the land-line market is substantiated by the higher rate of growth for land-line calls during Phase III. In contrast, NYNEX literature influenced more than half (59 percent) of all Phase III NYNEX users to call SmarTraveler with word of mouth reported by another 20 percent. Broadcast media sources (i.e., television and radio ads and mentions) collectively were the primary influence for only 13 percent of NYNEX users.

\section{PHASE IV MARKETING AND PRICING CHANGES}

As a result of the findings and recommendations from the Phase III evaluation, changes were made to marketing and pricing during Phase IV. The Phase III marketing was more successful among land-line phone users but overall did not result in large increases in awareness or trials. It was believed that cellular phone users still held the most potential, since NYNEX cellular phone users who received both free calls and direct marketing literature from NYNEX were the most avid users of the service while Cellular One subscribers represented almost twice as large a market yet remained virtually untapped. For these reasons, SmarTraveler pursued an arrangement with Cellular One that mirrored that with NYNEX. In July 1995 an agreement was reached between Cellular One and SmarTraveler in which Cellular One callers were offered free calls to SmarTraveler via the $* 1$ access number. A renewed marketing effort including radio ads, radio and TV mentions, and billboards was targeted to NYNEX and Cellular One cellular telephone subscribers highlighting the $* 1$ access number.

\section{IMPACT OF PHASE IV CHANGES}

During 1995 (Phase IV), SmarTraveler experienced a large increase in calls and users, largely among Cellular One users (which exhibited a tenfold increase in calls over a few months' time). Significant growth occurred during the second half of the year, after the marketing campaign and the new pricing arrangements were introduced.

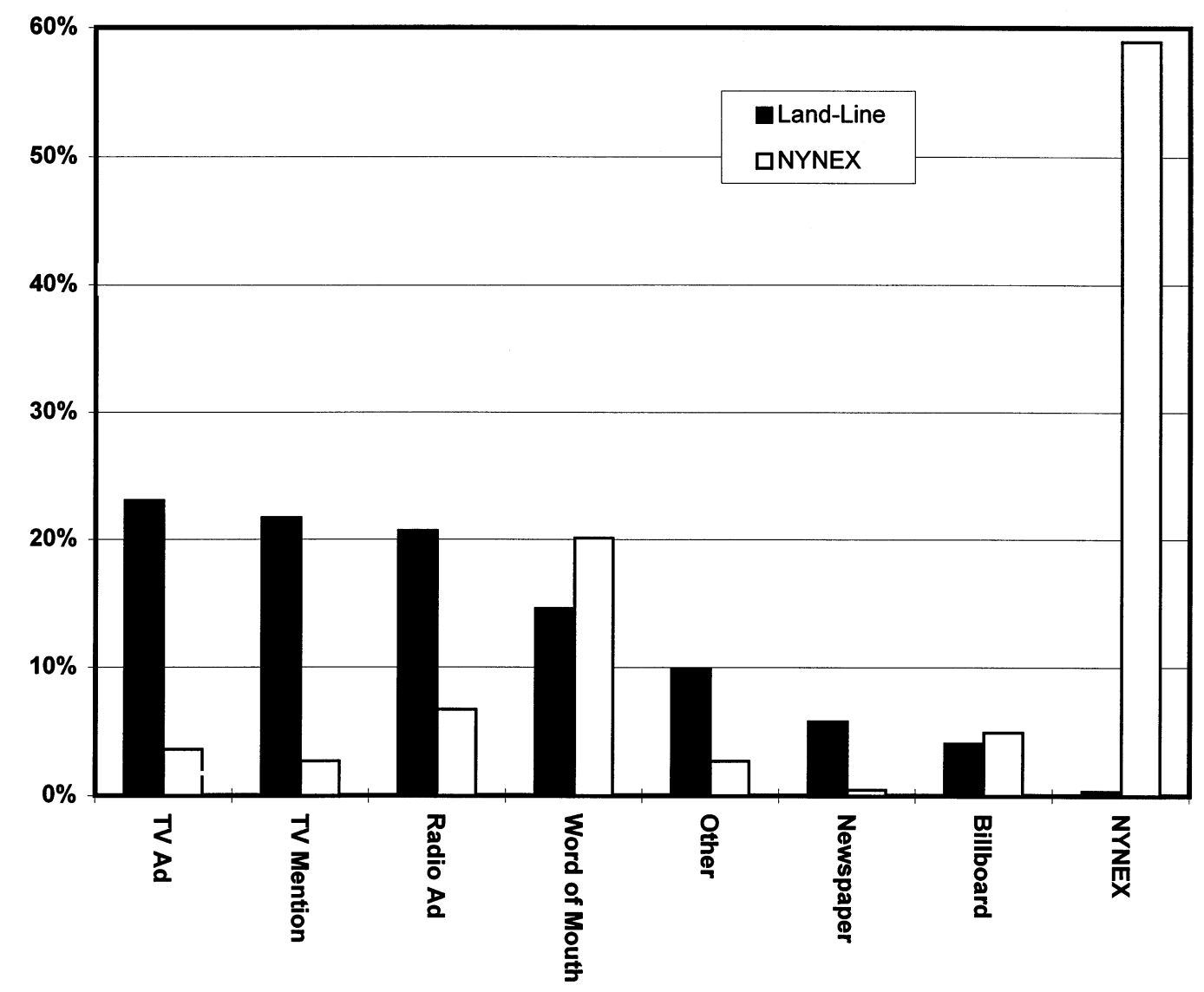

FIGURE 3 Marketing component that most influenced users to call. 
By October 1995, SmarTraveler was receiving nearly 57,000 weekly calls - more than twice the volume recorded a year earlier.

Because of this dramatic growth among cellular telephone users, a survey was conducted of a sample of cellular phone callers in November and December 1995 in order to learn more about their calling behavior. The following conclusions were drawn from that survey. On average, survey respondents estimated that they called SmarTraveler almost three times as often during the fall of 1995 as they did before the new pricing arrangements took effect. Moreover, most callers were shown to be quite price sensitive and indicated that they would stop using SmarTraveler if a service charge were imposed.

\section{Awareness and Trials}

About 44 percent of Cellular One callers learned about SmarTraveler during the summer and fall of 1995, compared with 36 percent of NYNEX users. Similarly, 45 percent of first-time callers learned about the service at this time, compared with 36 percent of repeat callers. Finally, of those who learned about SmarTraveler during summer and fall 1995, 54 percent were Cellular One callers, and 46 percent were NYNEX callers. Taken together, these findings suggest that the favorable pricing and marketing efforts directed at Cellular One subscribers attracted new callers during the second half of 1995 .

Most callers surveyed reported calling SmarTraveler within a month of learning about it ( 80 percent), and half said they called immediately. Overall, only 14 percent of all users waited longer than a month to call SmarTraveler. Callers most commonly reported waiting to call because of the charge or because they did not need the service. However, only a small number of callers cited specific reasons, which makes it difficult to draw valid conclusions.

\section{Calling Behavior}

Both the volume of calls and the number of users increased steadily through 1995. By October 1995, SmarTraveler was receiving nearly 57,000 weekly calls, which was more than twice the volume recorded in the comparable period in 1994. More than 20,000 people were estimated to be calling SmarTraveler on a weekly basis in October 1995-nearly three times the volume of users a year earlier.

Much of the increase in calls and users throughout 1995 can be attributed to the dramatic growth in calls from Cellular One phones. Between May and August 1995, the volume of Cellular One calls increased from less than 10 percent of the estimated weekly calls to more than 40 percent. By August, Cellular One callers (now using *1) were the largest group of SmarTraveler users at just over 40 percent of the calling population; NYNEX callers accounted for about a third of the users, and land-line callers made up the rest. In fact, starting in August 1995, the number of cellular telephone users exceeded land-line users for the first time. While weekly calls from cellular phones had previously outnumbered calls from landline phones, callers dialing 374-1234 had constituted the majority of weekly users until the Cellular One pricing change was implemented.

Because of this growth in cellular telephone usage, respondents in the cellular telephone survey were asked to estimate how often they called SmarTraveler before July 1995-before Cellular One callers could call for free-and currently (as of November 1995). Because these are recollections rather than documented behavior, they tend to reflect user perceptions of changes in calling activity. Before July 1995, respondents recalled calling SmarTraveler an average of 1.64 times per week on their cellular telephones-and most (69 percent) called less than once a week. NYNEX callers were far more likely than Cellular One users to be frequent callers before July; on average NYNEX users called 2.45 times a week, whereas Cellular One users called 0.82 times per week. About 59 percent of NYNEX users called less than once a week, compared with 80 percent of Cellular One users.

Calling frequency increased noticeably after July. Overall, respondents reported calling SmarTraveler 4.76 times a week during fall 1995, and the proportion calling less than once a week dropped to 21 percent. In November 1995, NYNEX users reported making 4.91 calls a week, and Cellular One callers nearly caught up, with 4.59 calls per week. Only 16 percent of Cellular One callers reported calling less than once a week in the fall, compared with 26 percent of NYNEX users.

\section{Sensitivity to Pricing Changes}

As stated earlier, SmarTraveler is currently offered as a free service as a result of public and private support. Callers from cellular telephones pay no charges whatsoever. Callers from conventional telephones pay the applicable charges for calls in area code 617; those with unlimited calling plans pay no per-charge calls for the service. In evaluations of previous phases of the SmarTraveler operational test, callers have proven quite sensitive to the suggestion of any kind of service charge for using SmarTraveler. The findings of the recent survey of cellular telephone callers are consistent with those earlier results.

The dramatic increase in average weekly calling rates among Cellular One callers between the early and latter parts of the year suggests that the availability of free calls had a noticeable influence on calling behavior. Though the estimated calling behavior diverged from the behavior recorded during the intercept surveys, the growth in recollected calling activity among Cellular One users is consistent with increased use under the new pricing scenario.

Callers were asked whether their employers paid their cellular telephone charges. The majority of callers (69 percent) paid for these charges themselves, which may be a factor in their overall resistance to paying a service charge for calling SmarTraveler.

To further test the influence of price on calling frequency, users were asked hypothetical questions intended to examine their sensitivity to changes in SmarTraveler pricing arrangements. First, callers were asked how they would react to two pricing scenarios: a monthly service charge of $\$ 2.50$ and a monthly service charge of $\$ 5.00$. Second, they were asked what they would do if their cellular telephone carrier introduced a service charge while another company did not.

Most callers indicated that they would stop calling SmarTraveler altogether under either of the two pricing scenarios (Figure 4). Overall, 61 percent said they would stop calling at a $\$ 2.50$ monthly service charge, and 70 percent would stop calling at the $\$ 5.00$ level. Estimates of average weekly frequency show a similar pattern. Among all cellular telephone callers-who reported calling an average of 4.76 times a week in November - the average respondent estimated that he or she would call SmarTraveler 1.84 times per week with a $\$ 2.50$ monthly charge and 1.32 times per week under a 


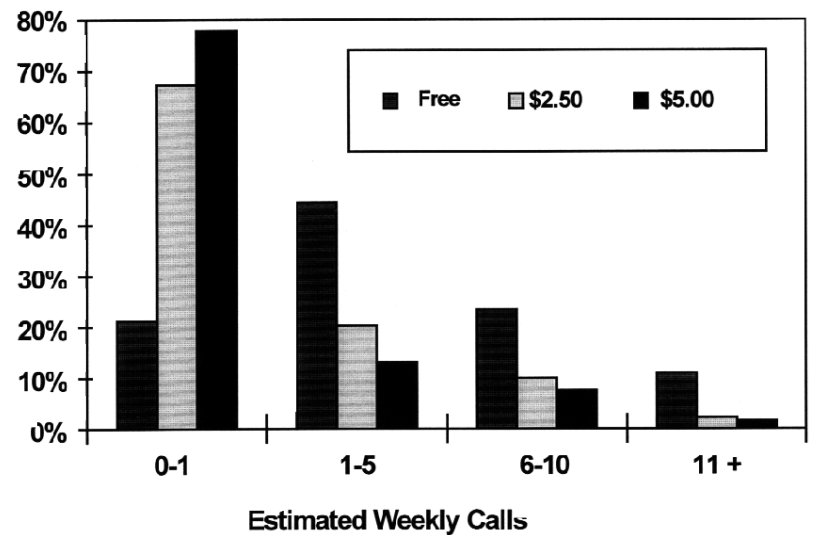

FIGURE 4 Calling frequency under different pricing scenarios.

$\$ 5.00$ monthly charge. Cellular One callers showed the greater sensitivity; they estimated that weekly calling would drop to 1.65 with a $\$ 2.50$ charge and to 0.94 with a $\$ 5.00$ charge. NYNEX callers were somewhat less sensitive, estimating calling rates of 2.01 and 1.66 , respectively. The greater sensitivity of Cellular One callers could be due to the shorter duration of their use of SmarTraveler or their greater expectation of reinstated calling charges.

To further examine their sensitivity to different types of pricing scenarios, callers were asked how they would react if their cellular telephone carrier imposed a service charge for using SmarTraveler while a competing carrier did not. Some 32 percent said they would switch phone carriers in order to continue using SmarTraveler for free, while about 35 percent said they would use SmarTraveler less often or not at all. Only 10 percent of callers did not anticipate changing their calling behavior under these circumstances, and almost 23 percent were not sure what they would do. On average, about 42 percent indicated a willingness to continue using SmarTraveler by either staying with their telephone carrier or switching companies. In comparison, about 35 percent said that they would stay with their present carrier but use SmarTraveler less, or not at all. Although anticipated behavior may not correlate exactly with observed behavior, callers are clearly showing resistance to the hypothetical imposition of service charges for using SmarTraveler.

\section{CONCLUSIONS}

The Phase III marketing program appears to have been only partly successful in increasing the public's level of awareness of SmarTraveler and in inducing trials. The marketing program reached a substantial share of its target audience ( 37 percent of all nonusers reported being exposed to one or more marketing activities), but no change was detected in background awareness levels between Phases II and III. It also appears that the marketing program was only partially successful in convincing its target market of SmarTraveler's superiority to radio and TV travel reports. Although 59 percent of the information seekers among the nonusers could distinguish SmarTraveler from conventional media traffic reports, a sizable minority (41 percent) believe that SmarTraveler provides exactly the same information as Channel $5 \mathrm{TV}$ or radio traffic reports. This is an important finding about the marketing program's effectiveness, since information seekers are an important potential market. Raising awareness and knowledge about how to access SmarTraveler will have little effect on increasing use of the service if those most likely to seek travel information believe that SmarTraveler provides only the same information they already obtain from other sources. Because SmarTraveler requires the user to seek out the information (by calling) while radio and TV travel reports are available routinely as a passive (or incidental) activity, it is very important for SmarTraveler to distinguish its unique advantages to potential users.

Initiating trials is extremely important to the continuing growth in the user population, but the media ads that composed much of the marketing program were the primary influence on only about onethird of all trials. In comparison, one-quarter cited word of mouth, (which is a by-product of the program but not the main focus). In a case in which the influence of word of mouth equals or approaches the influence of direct media, the effectiveness of the marketing campaign in promoting trials of the service might be questioned.

Although Phase III marketing induced greater response from land-line callers, cellular callers remained the most important market as evidenced by their higher participation and calling frequency. Although there were more subscribers to Cellular One phone service, the unfavorable pricing by Cellular One of calls to SmarTraveler resulted in very few calls to SmarTraveler. To tap this market, SmarTraveler negotiated free calls from Cellular One callers and resumed marketing targeted to cellular callers. Throughout 1995, cellular callers responded to SmarTraveler's targeted marketing campaign and the new Cellular One pricing arrangements to become an increasingly important part of the SmarTraveler market. As the survey has shown, more than half of current cellular callers first became aware of SmarTraveler during 1995, and most of these learned about the service in the latter half of the year.

Cellular telephone users call SmarTraveler frequently, with both NYNEX and Cellular One users reporting nearly daily calls. This is a significant increase from calling behavior before July 1995, when respondents remembered calling less than twice a week.

The service has continued to operate after the conclusion of the test period and is experiencing further growth in usage, particularly among cellular callers. However, it is important to note that most callers surveyed said they would stop using SmarTraveler if monthly service charges were introduced.

\section{ACKNOWLEDGMENTS}

The authors wish to acknowledge the guidance provided by Alicia Powell Wilson and Efi Pagitsas of the Central Transportation Planning Staff, the contributions of Joseph Brevard and Brian Barber of Planners Collaborative, and the surveyors and staff of Bernett Research Services, Inc. In addition, they acknowledge the cooperation, assistance, and comments provided by Stephen Crosby, David Stein, and Katy Miller Wallace of SmartRoute Systems.

Publication of this paper sponsored by Committee on Intelligent Transportation Systems. 